\title{
Development of a prognostic scoring system in patients with pneumonia requiring ventilator care for more than 4 days: a single-center observational study
}

\author{
Yeseul $\mathrm{Oh}^{1}$, Yewon Kang' ${ }^{2}$ Kwangha Lee ${ }^{1}$ \\ ${ }^{1}$ Division of Pulmonary, Allergy and Critical Care Medicine, Department of Internal Medicine, Pusan National University School of Medicine, Busan; \\ ${ }^{2}$ Department of Internal Medicine, VHS Medical Center, Busan, Korea
}

Background: The aim of the present study was to develop a prognostic model using demographic characteristics, comorbidities, and clinical variables measured on day 4 of mechanical ventilation (MV) for patients with prolonged acute mechanical ventilation (PAMV; MV for $>96$ hours).

Methods: Data from 437 patients (70.9\% male; median age, 68 years) were obtained over a period of 9 years. All patients were diagnosed with pneumonia. Binary logistic regression identified factors predicting mortality at 90 days after the start of MV. A PAMV prognosis score was calculating $\beta$-coefficient values and assigning points to variables.

Results: The overall 90 -day mortality rate was $47.1 \%$. Five factors (age $\geq 65$ years, body mass index $<18.5 \mathrm{~kg} / \mathrm{m}^{2}$, hemato-oncologic diseases as comorbidities, requirement for vasopressors on day 4 of MV, and requirement for neuromuscular blocking agents on day 4 of MV) were identified as prognostic indicators. Each factor was valued as +1 point and used to develop a PAMV prognosis score. This score showed acceptable discrimination (area under the receiver operating characteristic curve of 0.695 for mortality, $95 \%$ confidence interval $0.650-0.738$, $P<0.001$ ), and calibration (Hosmer-Lemeshow chi-square $=6.331$, with df 7 and $P=0.502$ ). The cutoff value for predicting mortality based on the maximum Youden index was $\leq 2$ (sensitivity, 87.5\%; specificity, 41.3\%). For patients with PAMV scores $\leq 1,2,3$, and $\geq 4$, the 90-day mortality rates were $29.2 \%, 45.7 \%, 67.9 \%$, and $90.9 \%$, respectively $(\mathrm{P}<0.001)$.

Conclusions: Our study developed a PAMV prognosis score for predicting 90-day mortality. Further research is needed to validate the utility of this score.

Key Words: mechanical ventilators; pneumonia; prognosis

\section{INTRODUCTION}

Advances in the early management of acute critically ill patients requiring ventilator care have led to long-term stays in the intensive care unit (ICU), which pose a high clinical and financial burden. Notably, critical care resources and facilities at university-affiliated tertiary care hospitals in Korea are typically more limited than those in North America and Europe $[1,2]$. Furthermore, patients' families or surrogates usually want to know a patient's medical status and likely clinical outcome if the patient is placed on mechanical ventilation (MV). Therefore, developing strategies to improve outcomes and establishing long-term care plans

\section{Original Article}

Received: September 22, 2020

Revised: December 24, 2020

Accepted: January 7, 2021

\section{Corresponding author} Kwangha Lee

Division of Pulmonary, Allergy and Critical Care Medicine, Department of Internal Medicine, Pusan National University School of Medicine, 179 Gudeok-ro, Seo-gu, Busan 49241, Korea

Tel: +82-51-240-7743

Fax: +82-52-245-3127

E-mail: jubilate@pusan.ac.kr

Copyright (@) 2021 The Korean Society of Critical Care Medicine

This is an Open Access article distributed under the terms of Creative Attributions Non-Commercial License (https:// creativecommons.org/li-censes/by-nc/4.0/ which permits unrestricted noncommercial use, distribution, and reproduction in any medium, provided the original work is properly cited. 
for patients at the early stage of MV is necessary.

Recent studies have described a novel subpopulation of patients on ventilator care that require MV for more than 96 hours, and these patients are referred to as "prolonged acute mechanical ventilation (PAMV)" cases $[3,4]$. This subpopulation is categorized as patients with "chronic critical illness" (CCI) [5] and is projected to increase over the next years [3]. If a PAMV case still shows severe illness on day 4 of MV, the long-term prognosis is likely to be poor. However, few studies have examined factors that affect the long-term prognosis of these patients.

Based on a report about factors that are prognostic for 28 day mortality [6], we hypothesized that some clinical variables on day 4 of MV may be useful for predicting the long-term prognosis of PAMV patients. Previous studies have suggested that demographic characteristics, body mass index (BMI), and comorbidities have prognostic value in patients with critical illness [7-10]. If these clinical parameters can be used to predict prognosis, it would be of great help to critical care physicians with respect to treatment planning at the early stage of ventilator care.

In the present study, we evaluated prognostic indicators that are useful for predicting the 90-day mortality of patients on PAMV. We also constructed a mortality prediction model (PAMV prognosis score) based on the prognostic factors. In addition, we compared the ability of this new PAMV prognosis score with that of two commonly used severity of illness scores (Acute Physiology and Chronic Health Evaluation [APACHE] II and Sequential Organ Failure Assessment [SOFA]) to predict mortality.

\section{MATERIALS AND METHODS}

In our study, the relevant medical, laboratory, and radiological data were extracted and used to fill out a case report form for each patient. These data were then analyzed. The study protocol was approved by the Institutional Review Board of Pusan National University Hospital (IRB No. 2003-012-088). Due to the retrospective and observational nature of our study, the need for informed consent from enrolled subjects or their surrogates was waived. The study had no impact on the treatment of enrolled patients.

\section{Study Design and Patient Selection}

This retrospective, observational study was conducted in the medical ICU of a 1,200 bed university-affiliated tertiary care hospital. This medical ICU has full cardiovascular facilities and close airway monitoring equipment, with a nurse-to-bed

\section{KEY MESSAGES}

- Developing strategies for the early stage of ventilator care are necessary to improve outcomes and establish a longterm care plan.

- A prognostic model based on older age, low body mass index, hemato-oncologic diseases as comorbidities, and clinical variables on day 4 of MV (requirement for vasopressors and neuromuscular blocking agents) is useful for predicting 90-day mortality in patients with pneumonia requiring ventilator care for more than 4 days.

ratio of 1:3. All subjects were managed according to therapeutic recommendations (based on a lung-protective ventilator strategy) [11].

Data from patients treated between March 1, 2008 and February 28, 2017 were evaluated retrospectively. All adult subjects ( $\geq 18$ years old) who were admitted to the medical ICU were assessed for eligibility; patients who were admitted to the coronary care unit were excluded. Patients diagnosed with pneumonia (community- and hospital-acquired pneumonia ([CAP] and [HAP] $)$ and receiving ventilator care for more than 96 hours (defined as PAMV) $[4,12]$ were included. Patients whose primary reason for ventilator care was not pneumonia were excluded, as were patients who received tracheostomy prior to hospital admission or less than 3 days after ICU admission; this is because early tracheostomy introduces bias when predicting prognosis (Figure 1). The definition of CAP and HAP was applied to enrolled patients; the definitions were based on those in the literature $[13,14]$.

\section{Data Collection}

Demographic and clinical data were obtained retrospectively

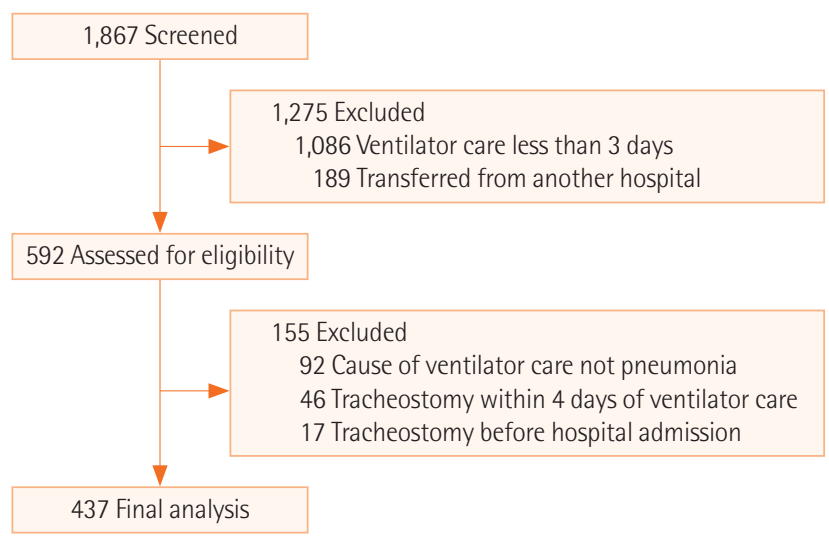

Figure 1. Flowchart of recruited and enrolled study participants. 
from electronic medical records; these data included age, sex, BMI, length of stay (LOS) in the ICU and hospital, and duration of MV. Severity of illness was measured using the APACHE II score; accompanying organ failure was assessed using the SOFA score $[15,16]$. The APACHE II and SOFA scores were calculated from laboratory and clinical data obtained within the first 24 hours of ICU admission. On admission, the underlying comorbidities of all enrolled subjected were gathered from medical records. In addition, Charlson's weighted index of comorbidities (WIC) was evaluated, as previous studies demonstrated the prognostic utility of this index in PAMV patients $[17,18]$.

Studies have reported that some clinical variables are prognostic factors in patients under ventilator care [6,19-22]. Therefore, the following laboratory and clinical data obtained on day 4 of MV were recorded: complete blood count, the lowest

Table 1. Clinical characteristics of survivors and non-survivors

\begin{tabular}{|c|c|c|c|c|}
\hline \multirow{2}{*}{ Variable } & \multirow{2}{*}{ Total $(n=437)$} & \multicolumn{2}{|c|}{ 90-Day mortality } & \multirow{2}{*}{ P-value } \\
\hline & & Survivor $(n=231)$ & Non-survivor $(n=206)$ & \\
\hline Male sex & $310(70.9)$ & 167 (72.3) & $143(69.4)$ & 0.528 \\
\hline Age (yr) & $68(18-91)$ & $66(18-91)$ & $71(21-91)$ & $<0.001$ \\
\hline Body mass index $\left(\mathrm{kg} / \mathrm{m}^{2}\right)$ & $21.5(11.0-45.8)$ & $21.8(14.7-36.2)$ & $21.0(11.0-45.8)$ & $<0.001$ \\
\hline ICU length of stay (day) & 18 (4-201) & $19(4-201)$ & $16(5-85)$ & 0.021 \\
\hline Hospital length of stay (day) & $29(6-432)$ & $40(6-432)$ & $24(6-90)$ & $<0.001$ \\
\hline Ventilator length of stay (day) & $13(4-198)$ & $13(4-198)$ & $13(4-79)$ & 0.003 \\
\hline APACHE II score ${ }^{a}$ & $19(3-44)$ & $18(3-38)$ & $21(6-44)$ & $<0.001$ \\
\hline SOFA score ${ }^{a}$ & $6(2-19)$ & $6(2-18)$ & $7(2-19)$ & 0.001 \\
\hline Charlson's weighted comorbidity index & $3(0-15)$ & $2(0-14)$ & $4(0-15)$ & $<0.001$ \\
\hline \multicolumn{5}{|l|}{ Underlying comorbidity ${ }^{b}$} \\
\hline Chronic lung disease & $190(43.5)$ & $84(36.4)$ & $106(51.5)$ & 0.002 \\
\hline Hemato-oncologic disease & $89(20.4)$ & $28(12.1)$ & $61(29.6)$ & $<0.001$ \\
\hline Neurologic disease & $80(18.3)$ & $51(22.1)$ & $29(14.1)$ & 0.035 \\
\hline Chronic kidney disease & $70(16.0)$ & $34(14.7)$ & $36(17.5)$ & 0.437 \\
\hline \multicolumn{5}{|l|}{ Clinical variable on fourth day of ventilator care ${ }^{c}$} \\
\hline Requirement of vasopressor & $200(45.8)$ & $86(37.2)$ & $114(55.3)$ & $<0.001$ \\
\hline Requirement of NMBA & $181(41.4)$ & $78(33.8)$ & $103(50.0)$ & 0.001 \\
\hline $\mathrm{PaO}_{2} / \mathrm{FiO}_{2}<200 \mathrm{~mm} \mathrm{Hg}$ & $169(38.7)$ & $77(33.3)$ & $92(44.7)$ & 0.018 \\
\hline Platelet count $<100 \times 10^{9} / \mathrm{L}$ & $151(34.6)$ & $64(27.7)$ & $87(42.2)$ & 0.002 \\
\hline Requirement of modialysis & $74(16.9)$ & $34(14.7)$ & $40(19.4)$ & 0.203 \\
\hline Multiple lobes involvement of chest X-ray ${ }^{d}$ & $368(84.6)$ & $188(81.7)$ & $180(87.8)$ & 0.085 \\
\hline Albumin $<3.5 \mathrm{~g} / \mathrm{dL}^{\mathrm{e}}$ & 346 (96.9) & $175(95.6)$ & $171(98.3)$ & 0.221 \\
\hline Uremia $(\mathrm{BUN}>7 \mathrm{mmol} / \mathrm{L})^{f}$ & $257(68.4)$ & $124(62.9)$ & $133(74.3)$ & 0.020 \\
\hline $\mathrm{pH}<7.35^{\mathrm{g}}$ & $106(24.7)$ & $39(17.3)$ & $67(32.7)$ & $<0.001$ \\
\hline Lactic acid >2.0 mmol/ $\mathrm{L}^{\mathrm{h}}$ & $105(40.9)$ & $45(34.1)$ & $60(48.0)$ & 0.031 \\
\hline Tracheostomy during ICU stay & $177(40.5)$ & $109(47.2)$ & $68(33.0)$ & 0.003 \\
\hline
\end{tabular}

Values are presented as number (\%) or median (range).

ICU: intensive care unit; APACHE: Acute Physiology and Chronic Health Evaluation; SOFA: Sequential Organ Failure Assessment; NMBA: neuromuscular blocking agents; BUN: blood urea nitrogen.

${ }^{a}$ All clinical data were calculated or obtained from medical records on the day of ICU admission; ${ }^{b}$ The four most common underlying diseases are presented. Chronic lung diseases included chronic obstructive pulmonary disease, interstitial lung disease, bronchiectasis, and tuberculosis-destroyed lung. Neurologic diseases included a previous history of cerebrovascular disease and neuromuscular disease; ' ${ }^{c}$ ll clinical data were calculated or obtained from medical records on day 4 of ventilator care; ${ }^{d}$ Data from 435 patients (survivors, 230; non-survivors, 205); ${ }^{e}$ Data from 357 patients (survivors, 183 ; non-survivors, 174); ${ }^{\text {DD }}$ ata from 376 patients (survivors, 197; non-survivors, 179); ${ }^{9}$ Data from 430 patients (survivors, 225; non-survivors, 205); ${ }^{h}$ Data from 257 patients (survivors, 132; non-survivors, 125). 
$\mathrm{PaO}_{2} / \mathrm{FiO}_{2}$ ratio, the need of vasopressors and hemodialysis, and unavoidable requirement of neuromuscular blocking agents (NMBAs) due to ventilator dyssynchrony (intermittent or continuous). We also recorded the following clinical variables on day 4 of MV, based on studies about severity and prognosis of CAP [23,24]: serum albumin, blood urea nitrogen (BUN), $\mathrm{pH}$ on arterial blood gas analysis, and simple chest radiograph. Lactic acid level was obtained on same day because accompanying septic shock would be important in predicting prognosis [25].

The ICU, in-hospital, and 90-day cumulative mortality rates after ventilator care were assessed. With respect to surviving patients discharged from hospital, the 90-day mortality rate was obtained from the National Health Insurance Service Database. The primary outcome measure was 90-day mortality.

\section{Statistical Analysis}

Continuous variables are expressed as the median (range) and were compared using the Student t-test or Mann-Whitney U-test, as applicable. Categorical variables are expressed as numbers (percentages) and were compared using the chisquare test or Fisher's exact test (for small numbers), as applicable. Stepwise logistic regression analysis was used to identify factors that are independent predictors of 90-day mortality. In addition, $\beta$-coefficients derived from multiple logistic regression were simplified and presented as natural numbers $>0$; these were calculated as the sum of the simplified $\beta$-coefficients, as previously described [19-22,26]. The prognostic model (PAMV prognosis score) was developed based on the sum of the $\beta$-coefficients. To predict 90-day survival, Kaplan-Meier estimates were stratified according to the score, and curves were compared using the log-rank test. The areas under the receiver operating characteristic curve (AUCs) were used to assess the discriminatory ability of the prognostic model. In addition, model calibration was assessed using the HosmerLemeshow test. The AUCs of all prognostic models were compared with those of the APACHE II and SOFA scores using the DeLong test, as described previously [27]. An optimal cutoff value was identified based on the maximum Youden's index [28]. The sensitivity (SS), specificity (SP), positive likelihood ratio (PLR), negative likelihood ratio (NLR), positive predictive value (PPV), and negative predictive value (NPV) for predicting mortality were determined. All tests were two-tailed and P-values $<0.05$ were considered statistically significant. All statistical analyses were performed using IBM SPSS ver. 24.0 (IBM Corp., Armonk, NY, USA) and MedCalc ver. 19.4 (MedCalc Software, Ostend, Belgium).

\section{RESULTS}

\section{Patient Characteristics}

During the study period, a total of 1,867 patients received ventilator care. After application of exclusion criteria, 437 were eligible for inclusion in the study (Figure 1). The ICU, in-hospital, and 90-day cumulative mortality rates were $35.2 \%(n=154)$, $39.1 \%(n=171)$, and $47.1 \%(n=206)$, respectively. Table 1 shows the baseline characteristics of the patient group and compares survivors and non-survivors at 90 days after ventilator care.

Table 2. Logistic regression analysis of mortality in all patients

\begin{tabular}{|c|c|c|c|c|c|}
\hline Variable & Unadjusted OR (95\% Cl) & P-value & Adjusted OR ${ }^{\mathrm{a}}(95 \% \mathrm{Cl})$ & P-value & $\beta$ value \\
\hline Age $\geq 65 \mathrm{yr}$ & $1.902(1.277-2.834)$ & 0.002 & $2.309(1.166-4.575)$ & 0.016 & 0.837 \\
\hline $\mathrm{BMI} \leq 18.5 \mathrm{~kg} / \mathrm{m}^{2}$ & 1.873 (1.129-3.107) & 0.015 & 3.823 (1.608-9.089) & 0.002 & 1.341 \\
\hline Hemato-oncologic diseases as comorbidity & $3.050(1.858-5.006)$ & $<0.001$ & $2.256(1.082-4.705)$ & 0.030 & 0.814 \\
\hline Requirement for vasopressor ${ }^{b}$ & $2.089(1.425-3.064)$ & $<0.001$ & $3.048(1.688-5.503)$ & $<0.001$ & 1.114 \\
\hline Requirement for $\mathrm{NMBA}^{\mathrm{b}}$ & 1.962 (1.333-2.885) & 0.001 & $2.756(1.479-5.133)$ & 0.001 & 1.104 \\
\hline Chronic lung diseases as comorbidity ${ }^{c}$ & $1.855(1.265-2.720)$ & 0.002 & & & \\
\hline Platelet count $<100 \times 10^{9} / \mathrm{L}^{\mathrm{b}}$ & $1.908(1.280-2.844)$ & 0.002 & & & \\
\hline $\mathrm{PaO}_{2} / \mathrm{FiO}_{2}<200 \mathrm{~mm} \mathrm{Hg}^{\mathrm{b}}$ & $1.614(1.095-2.378)$ & 0.016 & & & \\
\hline Uremia (BUN > $7 \mathrm{mmol} / \mathrm{L})^{b}$ & $1.702(1.093-2.650)$ & 0.019 & & & \\
\hline $\mathrm{pH}<7.35^{\mathrm{b}}$ & $2.315(1.473-3.639)$ & $<0.001$ & & & \\
\hline Lactic acid > $2.0 \mathrm{mmol} / \mathrm{L}^{\mathrm{b}}$ & 1.785 (1.080-2.950) & 0.024 & & & \\
\hline
\end{tabular}

OR: odds ratio; Cl: confidence interval; BMI: body mass index; NMBA: neuromuscular blocking agents; BUN: blood urea nitrogen.

${ }^{a}$ All values were included in the multivariate analysis using stepwise backward selection procedures (Hosmer-Lemeshow chi-square $=6.331, \mathrm{df}=7$; $\mathrm{P}=0.502) ;{ }^{b}$ All clinical data were calculated or obtained from medical records on day 4 of ventilator care; ${ }^{c}$ Chronic lung diseases included chronic obstructive pulmonary disease, interstitial lung disease, bronchiectasis, and tuberculosis-destroyed lung. 


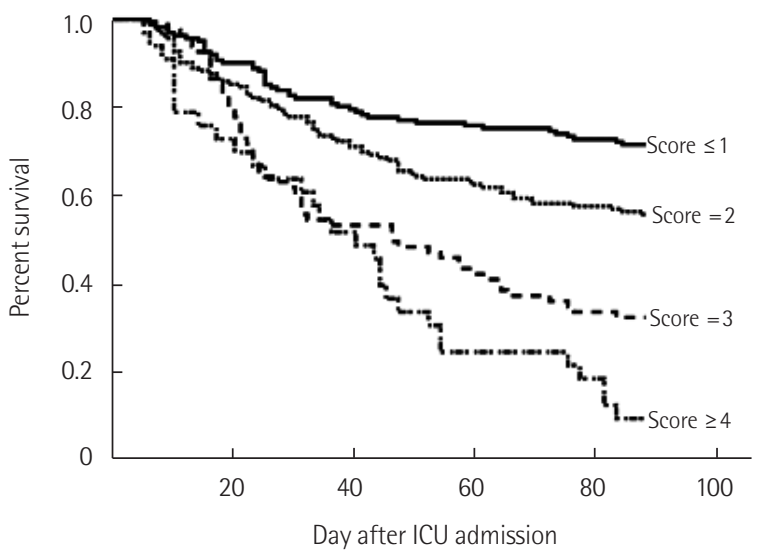

Figure 2. Kaplan-Meier survival curves, stratified according to the prolonged acute mechanical ventilation prognosis score (log-rank $<0.001)$. ICU: intensive care unit.

Non-survivors were older, had a lower BMI, and shorter ICU, hospital, and ventilator LOS than survivors. Non-survivors also had higher APACHE II and SOFA scores on ICU admission and Charlson's WIC than survivors. With respect to comorbidities, non-survivors had a significantly higher rate of chronic lung diseases (chronic obstructive pulmonary disease, interstitial lung disease, bronchiectasis, and tuberculosis-destroyed lung) and hemato-oncologic disease than survivors (Table 1). Comparison of clinical variables on day 4 of ventilator care revealed that non-survivors were more likely to require vasopressors and NMBAs than survivors. In addition, the percentages of patients with $\mathrm{PaO}_{2} / \mathrm{FiO}_{2}<200 \mathrm{~mm} \mathrm{Hg}$, platelet count $<100 \times 10^{9} / \mathrm{L}, \mathrm{BUN}>7 \mathrm{mmol} / \mathrm{L}, \mathrm{pH}<7.35$, and lactic acid $>2.0 \mathrm{mmol} / \mathrm{L}$ were significantly higher in non-survivors than among survivors (Table 1).

\section{Prognostic Factors Associated with 90-Day Mortality}

Univariate and multivariate analyses of risk factors predicting 90-day mortality are shown in Table 2. Multivariate analyses identified age $\geq 65$ years, BMI $<18.5 \mathrm{~kg} / \mathrm{m}^{2}$, hemato-oncologic diseases as comorbidities, and two clinical parameters on day 4 of ventilator care (requirement of vasopressors and NMBAs) as significant prognostic indicators of 90-day mortality.

\section{A Prognostic Model for Predicting 90-Day Mortality}

Based on the $\beta$-coefficients observed in multivariate analyses, a PAMV prognosis score was developed based on all of the factors; each factor was valued as +1 point. This score showed acceptable discrimination (AUC of 0.695 for mortality; $95 \%$ confidence interval $[\mathrm{CI}], 0.650-0.738 ; \mathrm{P}<0.001)$ and calibration (Hosmer - Lemeshow chi-square $=6.331$, with $\mathrm{df} 7$ and

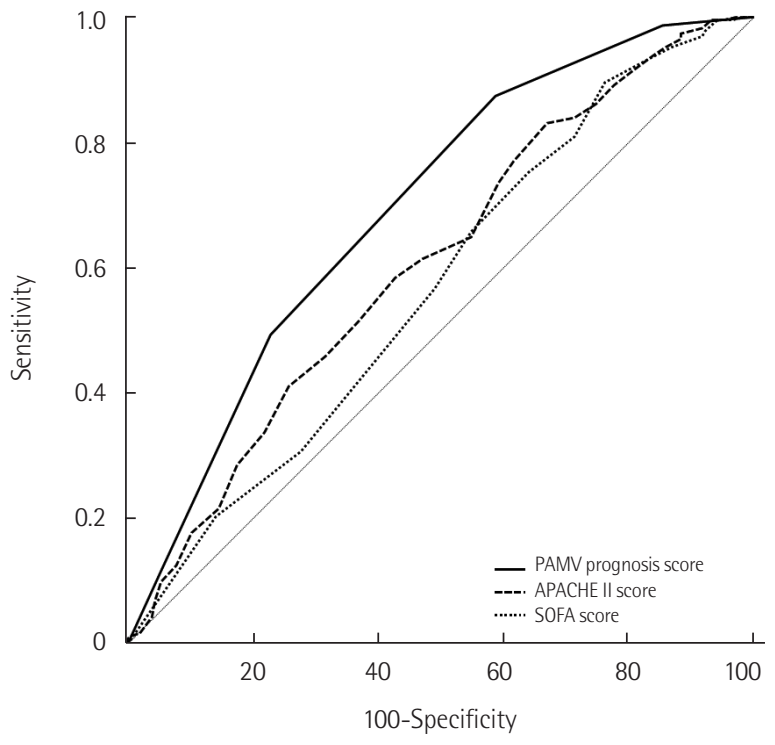

Figure 3. Comparison of receiver operating characteristic (ROC) curves for the prolonged acute mechanical ventilation (PAMV) prognosis score, Acute Physiology and Chronic Health Evaluation (APACHE) II score, and Sequential Organ Failure Assessment (SOFA) score for predicting 90-day mortality. For all patients, the areas under the ROC curve (AUCs) for the PAMV prognosis score, SOFA score, and APACHE II score were $0.695(95 \% \mathrm{Cl}, 0.650-0.738$; $\mathrm{P}<0.001), 0.608(95 \% \mathrm{Cl}, 0.561-0.654 ; \mathrm{P}<0.001)$, and $0.569(95 \%$ $\mathrm{Cl}, 0.521-0.616 ; \mathrm{P}<0.001)$, respectively. The AUC for the PAMV prognosis score was significantly higher than that of the other two scores ( $\mathrm{P}=0.009$ and $\mathrm{P}=0.001$, respectively). $\mathrm{Cl}$ : confidence interval.

$\mathrm{P}=0.502$ ) (Figure 2). The cutoff value for predicting mortality based on the maximum Youden index was $\leq 2$ (SS, 87.5\%; SP, 41.3\%; PLR, 2.2; NLR, 0.7; PPV, 62.5\%; and NPV, 74.6\%). KaplanMeier survival curves based on the model score were obtained for 90-day mortality relative to the score for this model (Figure 2 ). As the score increased, so did the mortality rate (score $\leq 1$, 2,3 , and $\geq 4=90$-day mortality rates of $29.2 \%, 45.7 \%, 67.9 \%$, and $90.9 \%$, respectively $)(\mathrm{P}<0.001)$.

For all patients, the AUCs for the PAMV prognosis, SOFA, and APACHE II scores were 0.695 (95\% CI, 0.650-0.738; P < 0.001), 0.608 (95\% CI, 0.561-0.654; $\mathrm{P}<0.001$ ), and 0.569 (95\% CI, 0.5210.616; $\mathrm{P}<0.001$ ), respectively. Comparison of the AUCs of this model with those of these two severity of illness scores revealed that the AUC of the PAMV prognosis score were significantly higher than those of the two scores $(\mathrm{P}=0.009$ and $\mathrm{P}=0.001$, respectively (Figure 3).

\section{DISCUSSION}

The present study evaluated prognostic factors in PAMV patients diagnosed with pneumonia in a medical ICU. The anal- 
ysis identified older age, being underweight, hemato-oncologic diseases as comorbidities, and clinical factors (requirement of vasopressors and NMBAs) on day 4 of MV as useful predictors of 90-day mortality. These indicators are readily applicable to these patients because they have been noted as prognostic factors in various reports in the field of critical care medicine $[6,7,9,20-22,29]$.

The results were used to develop a prediction model. The model showed good discrimination and calibration for predicting mortality. Based on the AUCs, the model was not inferior to the APACHE and SOFA scores obtained on the day of admission. Our PAMV prognosis model is objective and easy to extract, making it easy to link to electronic medical records to inform clinical care. Although this model should not override clinical judgment, the present study suggests that the model would help critical care physicians inform the patients' family or surrogates about the likely LOS in the ICU when a patient is placed on MV. However, the model requires further validation in large-scale multicenter studies.

The prevalence, long-term mortality, and costs of $\mathrm{CCI}$ are substantial [5]; therefore, prognostic models for predicting long-term outcomes have been developed using the clinical variables of patients on ventilator care. The ProVent score was validated to predict 1 -year mortality in patients requiring prolonged MV (defined as more than 21 days of MV) [21,30,31]. This score is based on four variables readily assessed on day 21 of MV: a requirement for dialysis, a requirement for vasopressors, age $\geq 50$ or $\geq 65$ years, and a platelet count $<150 \times 10^{9} / \mathrm{L}$. In addition, the extended ProVent model included comorbidities and laboratory data (albumin, bilirubin) [9]. Currently, the ProVent14 score is used to predict 1-year mortality in patients requiring MV for more than 14 days; this score is derived by assessing clinical variables on day 14 of MV. These variables include a requirement for dialysis, a requirement for vasopressors, age $\geq 50$ or $\geq 65$ years, a platelet count $<100 \times 10^{9} / \mathrm{L}$, and a non-trauma diagnosis [19,22]. A study of PAMV patients identified four variables on day 4 of MV (a requirement for vasopressors, a requirement for NMBAs, a requirement for dialysis, and a BMI $\leq 21 \mathrm{~kg} / \mathrm{m}^{2}$ ) as independent factors predictive of 28-day mortality [6]. The major advantage of the above-mentioned models is their simplicity, which means that they can be used in clinical practice and help inform management decisions regarding patients on MV. Herein, we included five clinical factors in developing the prognostic model, which is useful for predicting mortality. However, our PAMV prognosis model is not ideal for assessing prognosis in critically ill patients on MV, which raise several considerations. First, other clinical variables (such as $\mathrm{PaO}_{2} / \mathrm{FiO}_{2}<200 \mathrm{~mm} \mathrm{Hg}$, platelet count $<100 \times 10^{9} / \mathrm{L}$, albumin $<3.5 \mathrm{~g} / \mathrm{dL}, \mathrm{BUN}>7 \mathrm{mmol} / \mathrm{L}$, $\mathrm{pH}<7.35$, lactic acid $>2.0 \mathrm{mmol} / \mathrm{L}$, and serum sodium $\geq 145$ $\mathrm{mmol} / \mathrm{L}$ ) are reported as prognostic factors in critically ill patients [19,23-25,32]. Other research may find that these parameters are prognostic factors of PAMV patients. Second, clinical outcomes are affected by multiple extraneous factors, including the perspectives of the patient's family or surrogates, physician-family communications, and the utilization of medical resources. Third, critical care resources and facilities at university hospitals are limited, which also affects prognosis $[1,33]$. Therefore, developing new prediction models for patients requiring early stage ventilator care and for those requiring long-term ventilator care is necessary. In addition to clinical variables, socioeconomic, and ethical issues should be included in prognostic models and examined in large-scale multicenter studies.

The present study had several limitations. First, the study was conducted in the medical ICU of a single-center institution; therefore, the data may not be generalizable to other settings. Second, we hypothesized that the prognostic model would differ according to CAP and VAP; however, we could not accurately distinguish between these diagnoses due to the retrospective nature of the study. Third, other clinical variables such as organ dysfunction on day 4 of MV, or other comorbidities, could be considered for inclusion in the prognostic model; however, we found no such additional prognostic factors, which may be because of incomplete medical records. Finally, our study investigated prognostic factors for PAMV patients with a diagnosis of pneumonia; patients requiring PAMV due to other causes would need a different prognostic model. Further studies based on heterogeneous populations are needed to develop such prognostic models.

In conclusion, to the best of our knowledge, this study is the first to develop a prognostic model for predicting 90-day mortality in PAMV patients based on clinical variables on day 4 of MV, comorbidities, and BMI. Large-scale multicenter studies are needed to evaluate additional useful prognostic indicators and to develop new prognostic models with a higher power to predict long-term mortality. Such models will aid decisionmaking by critical care physicians at the early stage of MV.

\section{CONFLICT OF INTEREST}

No potential conflict of interest relevant to this article was reported. 


\section{ACKNOWLEDGMENTS}

This work was supported by a clinical research grant from $\mathrm{Pu}$ san National University Hospital (2020).

\section{ORCID}

Yeseul Oh

Yewon Kang

Kwangha Lee

https://orcid.org/0000-0002-9777-3258

https://orcid.org/0000-0002-7068-313X

https://orcid.org/0000-0001-9878-201X

\section{AUTHOR CONTRIBUTIONS}

Conceptualization: all authors. Data curation \& Formal analysis: YO, YK. Funding acquisition: KL. Methodology, Project administration, Visualization, \& Writing-original draft: all authors. Writing-review \& editing: KL.

\section{REFERENCES}

1. Kwak SH, Jeong CW, Lee SH, Lee HJ, Koh Y. Current status of intensive care units registered as critical care subspecialty training hospitals in Korea. J Korean Med Sci 2014;29:431-7.

2. Lim CM, Kwak SH, Suh GY, Koh Y. Critical care in Korea: present and future. J Korean Med Sci 2015;30:1540-4.

3. Zilberberg MD, Shorr AF. Prolonged acute mechanical ventilation and hospital bed utilization in 2020 in the United States: implications for budgets, plant and personnel planning. BMC Health Serv Res 2008;8:242.

4. Zilberberg MD, Kramer AA, Higgins TL, Shorr AF. Prolonged acute mechanical ventilation: implications for hospital benchmarking. Chest 2009;135:1157-62.

5. Kahn JM, Le T, Angus DC, Cox CE, Hough CL, White DB, et al. The epidemiology of chronic critical illness in the United States. Crit Care Med 2015;43:282-7.

6. Lee SH, Kim MJ, Jeong ES, Jo EJ, Eom JS, Mok JH, et al. Outcomes and prognostic factors in patients with prolonged acute mechanical ventilation: a single-center study in Korea. J Crit Care 2015;30:1016-20.

7. Shankar-Hari M, Ambler M, Mahalingasivam V, Jones A, Rowan K, Rubenfeld GD. Evidence for a causal link between sepsis and long-term mortality: a systematic review of epidemiologic studies. Crit Care 2016;20:101.

8. Wang S, Liu X, Chen Q, Liu C, Huang C, Fang X. The role of increased body mass index in outcomes of sepsis: a systematic review and meta-analysis. BMC Anesthesiol 2017;17:118.

9. Park YR, Lee JS, Kim HJ, Hong SB, Lim CM, Koh Y, et al. Modi- fication of the prolonged mechanical ventilation prognostic model score to predict short-term and 1-year mortalities. Respirology 2019;24:179-85.

10. Ni YN, Luo J, Yu H, Wang YW, Hu YH, Liu D, et al. Can body mass index predict clinical outcomes for patients with acute lung injury/acute respiratory distress syndrome? A meta-analysis. Crit Care 2017;21:36.

11. Acute Respiratory Distress Syndrome Network, Brower RG, Matthay MA, Morris A, Schoenfeld D, Thompson BT, et al. Ventilation with lower tidal volumes as compared with traditional tidal volumes for acute lung injury and the acute respiratory distress syndrome. N Engl J Med 2000;342:1301-8.

12. Zilberberg MD, Luippold RS, Sulsky S, Shorr AF. Prolonged acute mechanical ventilation, hospital resource utilization, and mortality in the United States. Crit Care Med 2008;36:72430.

13. Hospital-acquired pneumonia in adults: diagnosis, assessment of severity, initial antimicrobial therapy, and preventive strategies. A consensus statement, American Thoracic Society, November 1995. Am J Respir Crit Care Med 1996;153:171125.

14. Musher DM, Thorner AR. Community-acquired pneumonia. N Engl J Med 2014;371:1619-28.

15. Knaus WA, Draper EA, Wagner DP, Zimmerman JE. APACHE II: a severity of disease classification system. Crit Care Med 1985;13:818-29.

16. Vincent JL, Moreno R, Takala J, Willatts S, De Mendonça A, Bruining $\mathrm{H}$, et al. The SOFA (Sepsis-related Organ Failure Assessment) score to describe organ dysfunction/failure. On behalf of the Working Group on Sepsis-Related Problems of the European Society of Intensive Care Medicine. Intensive Care Med 1996;22:707-10.

17. Charlson ME, Pompei P, Ales KL, MacKenzie CR. A new method of classifying prognostic comorbidity in longitudinal studies: development and validation. J Chronic Dis 1987;40:37383.

18. Song SE, Lee SH, Jo EJ, Eom JS, Mok JH, Kim MH, et al. The prognostic value of the Charlson's comorbidity index in patients with prolonged acute mechanical ventilation: a single center experience. Tuberc Respir Dis (Seoul) 2016;79:289-94.

19. Kim WY, Jo EJ, Eom JS, Mok J, Kim MH, Kim KU, et al. Validation of the prognosis for prolonged ventilation (ProVent) score in patients receiving 14 days of mechanical ventilation. J Crit Care 2018;44:249-54

20. Roh J, Jo EJ, Eom JS, Mok J, Kim MH, Kim KU, et al. Factors predicting long-term survival of patients with sepsis on arrival at the emergency department: a single-center, observation- 
al study. Medicine (Baltimore) 2019;98:e16871.

21. Carson SS, Kahn JM, Hough CL, Seeley EJ, White DB, Douglas IS, et al. A multicenter mortality prediction model for patients receiving prolonged mechanical ventilation. Crit Care Med 2012;40:1171-6.

22. Hough CL, Caldwell ES, Cox CE, Douglas IS, Kahn JM, White DB, et al. Development and validation of a mortality prediction model for patients receiving 14 days of mechanical ventilation. Crit Care Med 2015;43:2339-45.

23. Mandell LA, Wunderink RG, Anzueto A, Bartlett JG, Campbell GD, Dean NC, et al. Infectious Diseases Society of America/American Thoracic Society consensus guidelines on the management of community-acquired pneumonia in adults. Clin Infect Dis 2007;44(Suppl 2):S27-72.

24. Charles PG, Wolfe R, Whitby M, Fine MJ, Fuller AJ, Stirling R, et al. SMART-COP: a tool for predicting the need for intensive respiratory or vasopressor support in community-acquired pneumonia. Clin Infect Dis 2008;47:375-84.

25. Rhodes A, Evans LE, Alhazzani W, Levy MM, Antonelli M, Ferrer $\mathrm{R}$, et al. Surviving sepsis campaign: international guidelines for management of sepsis and septic shock: 2016. Intensive Care Med 2017;43:304-77.

26. Carson SS, Garrett J, Hanson LC, Lanier J, Govert J, Brake MC, et al. A prognostic model for one-year mortality in patients requiring prolonged mechanical ventilation. Crit Care Med 2008;36:2061-9.

27. DeLong ER, DeLong DM, Clarke-Pearson DL. Comparing the areas under two or more correlated receiver operating char- acteristic curves: a nonparametric approach. Biometrics 1988; 44:837-45.

28. Perkins NJ, Schisterman EF. The inconsistency of "optimal" cutpoints obtained using two criteria based on the receiver operating characteristic curve. Am J Epidemiol 2006;163:670-5.

29. Wallace SK, Rathi NK, Waller DK, Ensor JE Jr, Haque SA, Price $\mathrm{KJ}$, et al. Two decades of ICU utilization and hospital outcomes in a comprehensive cancer center. Crit Care Med 2016;44:92633.

30. Leroy G, Devos P, Lambiotte F, Thévenin D, Leroy O. One-year mortality in patients requiring prolonged mechanical ventilation: multicenter evaluation of the ProVent score. Crit Care 2014;18:R155

31. Mok JH, Kim YH, Jeong ES, Eom JS, Kim MH, Kim KU, et al. Clinical application of the ProVent score in Korean patients requiring prolonged mechanical ventilation: a 10-year experience in a university-affiliated tertiary hospital. J Crit Care 2016;33:158-62.

32. Azevedo do Carmo T, Bonifácio Brige Ferreira I, Carvalho de Menezes R, Piñeiro Telles G, Lisboa Otero M, Arriaga MB, et al. Derivation and validation of a novel severity scoring system for pneumonia at ICU admission. Clin Infect Dis 2020 Mar 8 [Epub]. Available from: https://doi.org/10.1093/cid/ ciaal83.

33. Kim JH, Hong SK, Kim KC, Lee MG, Lee KM, Jung SS, et al. Influence of full-time intensivist and the nurse-to-patient ratio on the implementation of severe sepsis bundles in Korean intensive care units. J Crit Care 2012;27:414. 